\title{
"Killing Two Birds with One Stone"? A Case Study of Development Use of Drones
}

\author{
Wang, Ning
}

\begin{abstract}
With the rise of the "humanitarian drone" in recent years, drones have become one of the most controversial public interest technologies that have gained increasing media attention. It is worth noting that, although there is a perception in the aid sector that drones hold the promise to reinvent the health supply logistics, to date, routine drone delivery is still relatively new and largely unproven. This paper presents a recent field study conducted in 2019, where drones were deployed in Malawi to help address the last mile challenge in medical supply delivery, and where a noticeable mentality of "killing two birds with one stone" around the attempt of using drones in resource-poor settings is observed. The objective of the paper is to shed light, through a real-world case study and from the ethical perspective, on the impacts of implementing such a systemic change in the existing health supply chain systems. As conclusion, a call for more reflexive approaches for the critical examination, as well as more structured guidance for the responsible evaluation, of medical cargo drones is raised.
\end{abstract}

DOI: https://doi.org/10.1109/ISTAS50296.2020.9462187

Posted at the Zurich Open Repository and Archive, University of Zurich

ZORA URL: https://doi.org/10.5167/uzh-204596

Conference or Workshop Item

Accepted Version

Originally published at:

Wang, Ning (2020). "Killing Two Birds with One Stone"? A Case Study of Development Use of Drones. In: 2020 IEEE International Symposium on Technology and Society (ISTAS), Tempe, AZ, USA, 12 November 2020 - 15 November 2020. IEEE, 339-345.

DOI: https://doi.org/10.1109/ISTAS50296.2020.9462187 


\title{
"Killing Two Birds with One Stone"?
}

\section{A Case Study of Development Use of Drones}

\author{
Ning Wang \\ Institute of Biomedical Ethics and History of Medicine (IBME) \\ University of Zurich \\ Zurich, Switzerland \\ ning.wang@ibme.uzh.ch
}

\begin{abstract}
With the rise of the "humanitarian drone" in recent years, drones have become one of the most controversial public interest technologies that have gained increasing media attention. It is worth noting that, although there is a perception in the aid sector that drones hold the promise to reinvent the health supply logistics, to date, routine drone delivery is still relatively new and largely unproven. This paper presents a recent field study conducted in 2019, where drones were deployed in Malawi to help address the last mile challenge in medical supply delivery, and where a noticeable mentality of "killing two birds with one stone" around the attempt of using drones in resource-poor settings is observed. The objective of the paper is to shed light, through a real-world case study and from the ethical perspective, on the impacts of implementing such a systemic change in the existing health supply chain systems. As conclusion, a call for more reflexive approaches for the critical examination, as well as more structured guidance for the responsible evaluation, of medical cargo drones is raised.
\end{abstract}

Keywords—public interest technology; humanitarian drone; medical supply delivery; medical cargo drone; health supply chain system.

\section{INTRODUCTION}

With the rise of the "humanitarian drone" in recent years, drones have become one of the most controversial public interest technologies [1]. Although using drones to deliver medicines in hard-to-reach areas has gained increasing media attention, routine drone delivery is still relatively new and largely unproven [2][3]. Since 2016, Zipline has operated drones for the Government of Rwanda, delivering up to 3 liters of blood within 30 minutes to health facilities that request it on demand [4]. In 2017, the Government of Malawi (GoM) established the Humanitarian Drone Testing Corridor (hereafter referred to as the Drone Corridor), in collaboration with a specialized UN agency, where studies on using drones to facilitate last mile delivery (LMD) and integrating drones in an optimized health supply chain system in low-resource settings, were implemented [5]. In 2019, several other companies received approval to conduct routine flights or test deliveries, including transports of laboratory specimens in the USA by Matternet, and deliveries of over-the-counter medicines in Australia by Alphabet's Wing [6][7]. It is worth noting that although there is a perception in the aid sector that drones hold the promise to reinvent the health supply logistics, to date, Zipline is the only example of routine drone delivery of medical supplies in an actual operational environment [8].

The principle of public interest draws original inspiration from the concepts of newsworthiness and the public good [9]. The tendency of framing technology as a panacea for the aid sector potentially leads to the technological fantasy, where "newness" and "newsworthiness" are hyped up while the public good and public interest de-prioritized [1][10]. As the demand and complexity of aid programs in challenging conditions continue to expand, populations who live in remote locations experience significant obstacles to receive aid supplies, widening the gap of equitable access among the most vulnerable. These barriers give drones new purposes beyond their military origins, whereby they can be used to supplement the existing health supply chain systems to address the LMD challenge [11]. Since "do no harm" is not only about minimizing physical and material well-being, but as well maximizing the public good, one important evaluative criterion is whether the expected benefits of using drones to assist development work outweigh the potential risks over the long run [12][13].

This paper presents a recent field study conducted in Malawi during November 2019 and February 2020, where semi-structured qualitative interviews were carried out by the author (detailed research methods regarding data collection and analysis is documented in a forthcoming paper). In this study, drones were deployed to help address the medical supply delivery challenges in Malawi. The objective of the paper is to shed light, through a real-world case study, on risks and potential benefits of implementing such a systemic change from the ethical perspective. The paper is structured in three parts: 1) a medical delivery drone project operated in Malawi is introduced to set the context; 2) a general analysis of the field study is examined from the ethical perspective, whereby a noticeable mentality of "killing two birds with one stone" around the attempt of using drones to tackle health logistics challenges is observed, leading to the discussion that while the drone technology is portrayed as providing greater public good, its various limitations still await to be overcome through continued flight tests and feasibility studies; and 3) as conclusion, the author calls for a more reflexive approach in adopting new technology and implementing innovation policy in the aid sector.

\section{A Drone DELIVERY ProJeCt}

\section{A. Why Were Drones Proposed?}

$\mathrm{Y}$ is a government donor agency in the area of public health and international aid. By mandate, $\mathrm{Y}$ is involved with selecting, procuring, and delivering medications for infectious diseases, such as HIV/AIDS, as part of its global health initiatives, especially in countries in Africa, Asia and Latin America. It typically works in countries that are limited in resources in terms of personnel, finance, expertise, and infrastructure on the ground. In this context, it was perceived that the drone technology had unrealized potential to leapfrog poor infrastructure, and that there might be opportunities to use drones to provide uninterrupted supplies of health commodities, including drug delivery and lab sample collection and transportation. Y, therefore, decided to test if they could improve disease control using drones, and how they could undertake and manage drone operations in challenging environments. To this end, Y secured funding to 
explore the feasibility and demonstrate the applicability of drones through a pilot study. As the funding for this particular activity was earmarked exclusively for HIV/AIDS, where a larger budget was available, HIV/AIDS was chosen to be the focused health challenge area.

$\mathrm{C}$ is a US-based for-profit company, and one of the longterm implementers of $\mathrm{Y}$, with a team that aims at providing global supply chain services. Specifically, in collaboration with its industry and consultancy partners, $\mathrm{C}$ offers technical assistance to promote efficient health supply chain solutions using commercial processes. $\mathrm{Y}$ contracted $\mathrm{C}$ to design an activity utilizing drones to enhance system responsiveness and improve health outcomes related to HIV diagnosis. C started with several initiatives and produced a set of deliverables, one of which was a landscape analysis of drone applications in the development context. Since Y has priority countries and preferred the pilot location to be in Africa, where public health needs would match their funding priorities, $\mathrm{C}$ selected several countries in Africa and conducted site visits and feasibility assessments. As Y intended to answer the question of how to integrate drones in the national health supply chains, the envisioned activity focused on addressing the LMD challenge by improving the existing lab sample collection and transportation process. As a result, the goal of the project became one of matching a potential technology with a country in Africa where accessibility to hard-to-reach areas poses a challenge to its healthcare system.

As the body of knowledge expanded, $\mathrm{Y}$ gained better understanding of the regulatory environment, as well as how it would influence their project goals. Although it seemed that, across Africa, there is an overall openness towards drones with a generally open attitude to new technology and a largely open airspace to flying objects including unmanned aircrafts - the market drivers for drones varied from country to country [8]. For instance, in terms of logistics, DRC has one of the least developed infrastructure footprints in Africa, and drones could fill a need by flying over rainforest and terrain that surface vehicle cannot drive through due to a lack of roads. However, the Government of DRC is sensitive to unmanned aircrafts as a result of continued armed conflicts in the region, making it more complicated to carry out testing activities in DRC [14]. Given that the activity was designed to demonstrate the usefulness of drones for medical delivery in low-resource settings, Y needed to find a country that was "drone friendly". Eventually, all considerations taken together, it was decided that Malawi would be an ideal testing ground. What they then needed is finding a location within Malawi that would suit the proposed activity.

\section{B. Who Was Involved in the Project?}

In 2017, C kicked off the drone project. While looking for suitable locations to carry out the proposed activity, $\mathrm{C}$ noted that there was little publicly available information about how to procure drone services to assist development work. As not every actor had the resource and opportunity to conduct drone testing activities, $\mathrm{C}$ produced a guide that not only set the basis for their own procurement need, but could potentially also benefit the wider drone community. $\mathrm{C}$ then invited drone operators to bid and selected candidates based on their technical capacity. At the end of that process, the proposed activity was subcontracted with $\mathrm{D}$, a Europe-based drone manufacturer, focusing on the development, production and distribution of highly efficient industrial unmanned aerial vehicles (drones). Under the project management of C, D was responsible for the technical operation of the drones, whereby the two islands on Lake Malawi would be connected to the district hospital on the lakeshore of the mainland, which has road connection to the Central Molecular Laboratory of Malawi, and from where the formal supply chain exists and operates. As an external subcontractor, D provides the drones, the pilots, and some ground staff. In particular, the operation tasks range from providing technical assistance including flight planning and monitoring, to flying and maintaining high level control of the drone; while the project coordination, such as communicating with the GoM, setting the scene, and acquiring approvals, is organized directly by $\mathrm{C}$.

According to the Ministry of Health $(\mathrm{MoH})$ of Malawi, drones were not unfamiliar to them as already in 2016, they were approached by E, a specialized UN agency, proposing to explore the of use drones for health commodity delivery. In this feasibility study, E collaborated with a local NGO to transport stimulated dried blood samples between health facilities and laboratories for HIV diagnosis in infants [15]. Having worked on the ground for decades in Malawi, E has a comprehensive understanding about the Malawian context, and sought early support from the Department of Civil Aviation (DCA) of Malawi. From the $\mathrm{MoH}$ perspective, since E was their trusted long-term partner in terms of health system strengthening, and the proposal of quality health service provision was already in their strategic planning, they were open to learn how useful drones might be in bridging the service gaps. However, they did not have the resources required to operate drones in Malawi and would rely on donor funds. From the DCA perspective, they saw the potentials of the drone technology but needed to make sure that, first of all, their health sector would indeed benefit from the use of the drone technology; and secondly, the aviation sector would actually be able to build the technical capacity to implement and administer drones in Malawi in the long run.

Shortly after this feasibility study, E had an initiative to create a technology sandbox to safely facilitate drone testing activities and feasibility studies in Malawi. According to E, they approached the $\mathrm{MoH}$, the DCA, and the Ministry of Transport and Public Works with this proposal, to discuss the creation of the aforementioned Drone Corridor. Considering the potential benefits of the drone technology for Malawi, and that there is a small airport nearby Lilongwe that is away from manned aircrafts and no longer in use, they decided to designate it specifically to drones and launched the Malawi Drone Corridor. As the management of the Corridor exceeded the existing capacity of the DCA, they needed a partner with technical knowledge to help administer it. E provided capacity building support to the DCA personnel through technical trainings in the USA, and received an initial two-year mandate to manage the Corridor on their behalf, which was later extended to June 2021. Since then, E was officially involved in the humanitarian drone projects taking place in Malawi. In terms of process, the DCA is the authority for all flight approvals, prior to which, drone operators seeking approvals need to submit their applications to $\mathrm{E}$ and demonstrate their technical capacities inside the Drone Corridor. E helps the DCA with the evaluation of applications through an internal assessment process. In the case of acceptance, the DCA is informed to review the supporting documentation including licenses and insurance etc., and grants the final approval. 


\section{How Were Drones Introduced?}

According to $\mathrm{C}$, to ensure favorable operational conditions for this project, they conducted two scoping trips to Malawi in 2018 , including visits to potential sites referred to them by incountry partners. The goal was to verify the level of need for a range of possible drone activities at local health facilities. As results, some prime use cases were identified, and a concept of operations was developed. As the initial phase, two districts in the Northern Region of Malawi - Nkhata Bay and Likoma - were chosen to be the areas of pilot operations. The Nkhata Bay District has a district hospital, which was set as the primary base of operations. The Likoma District includes the island of Likoma, where a hospital managed by the Christian Health Association of Malawi (CHAM) is located, and its neighboring small island Chizumulu, where a health center was located. The drone operation was designed to initially take place between the Nkhata Bay district hospital and the Likoma Island hospital (managed by CHAM), and was later expanded to also include deliveries to the Chizumulu Island health center (managed by the $\mathrm{MoH}$ ). The service was expected to benefit both districts - as the health facilities on both islands need to transport lab samples to the mainland for analysis, while Nkhata Bay has remote areas that the existing health logistics cannot easily reach.

From when the project site in Malawi was identified to getting the final flight approvals, it took nearly a year where $\mathrm{C}$ had rounds of stakeholder meetings with the GoM to discuss the project plans, and to schedule the drone test flights. According to C, in November 2018, the DCA granted them the first approval to undertake the proposed activity in the identified area of operations. In April 2019, a stakeholder meeting was held in Lilongwe, including key representatives from the $\mathrm{MoH}$, the DCA, the District Health Officers of Nkhata Bay and Likoma, as well as E. During this meeting, approval was confirmed at the central level, upon which the revised activity plan was reported to the Traditional Authorities in the two involved districts. District Executive Council meetings were then held at the local level to review the activity design. In June 2019, final approvals were granted by the DCA through a formal issuance of a Remotely Piloted Aircraft Operations Certificate, valid till the end of 2019. As expressed by $\mathrm{C}$, they were the first entity that received approvals to carry out medical supply delivery activities using drones in Malawi, beyond visual line of sight (BVLOS) and outside the Drone Corridor.

$\mathrm{C}$ noted that the core idea of the pilot operation was to transport essential medical commodities from the district hospital in Nkhata Bay to the health facilities in Likoma and Chizumulu, and transport lab samples from these facilities to testing labs in the mainland, back and forth over Lake Malawi. Whereas it takes the boat about 6-8 hours for a oneway trip, it takes just an hour with the drone. Most lab samples are related to TB and HIV, including dried blood samples and sputum. According to the operating procures, once samples are collected from the two islands, they are sent timely to the district hospital in Nkhata Bay, which are then sent to the nearby molecular lab in Mzuzu for analysis. Then after the samples are processed, a report of the test results is sent by the drone back to the health facilities in the two islands. The tests can be done on an ad hoc basis, and depending on the demand, the drone can operate daily with multiple flights. In addition to lab samples, the drone can also transport drugs, vaccines, blood products, small medical devices, and documents. For goods that require a cold chain, there is Styrofoam insulation fitted inside the cargo box of the drone to keep the temperature low during the flight.

With respect to the communities involved in the project, as reported by interview participants of this field study, they were primarily the villagers living in the two involved districts. These villagers had no prior exposure to drones but were made aware of their coming, thanks to a large-scale community sensitization campaign. The campaign was led by E, who had extensive experience on communications for development, and was implemented in cooperation with the Ministry of Information and Civic Education, who was the key driver of the community sensitization activity. The campaign took place in the forms of theatrical plays and fireside chats to demonstrate what a drone was and how it flew, and was broadcasted on radio and TV [5]. E dedicated funds to educate the community members about the drone technology, and to reassure the communities that they would benefit from the drone deliveries. The district health promotion teams, who are the frontline health service providers trusted by local communities, were also utilized to assist the community sensitization at the local level. As nothing written from the community members was required, the Executive Council of the involved districts gave the approval on their behalf.

\section{How Were Drones Operated?}

According to the technical staff participated interviews in this filed study, prior to moving to the districts, C and D spent two weeks in the Drone Corridor to demonstrate their technical capabilities to conduct the proposed activity in a controlled and monitored environment, including flight, software, and communication solutions. In June 2019, upon receiving the aforementioned Operations Certificate, the project team moved to Likoma Island, where test flights were conducted with the drone flying one direction on incremental distances. In July 2019, the project entered into full operation between Nkhata Bay and Likoma Island, and later expanded to also include Chizumulu Island. As the then-Chief Flight Operator at D reported, between July and October 2019, over 200 flights operated for more than 90 hours, and about $45 \mathrm{~kg}$ of medical commodities were delivered over about $30,000 \mathrm{~km}$ flight route, making it almost 4-months of routine deliveries with reduced sample turnaround time of 0-4 weeks.

The operations were led by $\mathrm{D}$, through a technical team consisting of flight operators and remote pilots - the former responsible for mission planning, clearing the flights with local air traffic control, monitoring the ground control station during flights, and documenting operation data including altitude, speed, and battery level; and the latter responsible for additional safety measures during take-off and landing, as well as taking over manual control when necessary. According to the technical staff, there were two drones imported to Malawi - one for full-time use and the other for back up and training purposes. The operational range in the local environment was about $86 \mathrm{~km}$ for a one-way flight with full battery load, with a 13-liter payload cargo box below, or approximately $35 \mathrm{~km}$ for a two-way drop-off-only flight by hovering at cargo releasing site without landing. The flights were fully automated between take-off and landing, i.e., the so-called BVLOS flight. With the assistance of the ground staff, D had seven checklists to go through before each flight to ensure safety. Weather conditions were continuously assessed, and the drone was permanently monitored from the ground station.

Regarding the ground control station, in the mainland, the Nkhata Bay district hospital was set as the project base, where 
the drone was kept and an outdoor take-off site was located. On Likoma Island, the drone port was set at the airport outside the hospital. According to the operating procedures, when a need is identified at the local health facilities on the islands, trained health worker will initiate communications with their counterparts at the district hospital in the mainland. Then the needed products will be loaded onto the drone in the mainland, and the local health facilities will be notified of an estimated time of arrival of the drone. Once the drone will land on the islands, the trained health worker can remove the cargo box from the drone and reload it with lab samples or other materials that need to be transported to the mainland. For sample pickup, the lab technicians package the samples at the local health facilities and pass them over to the trained health worker who will bring the samples to the take-off location and load them onto the drone. Once loaded and ready for return, the local health worker can communicate with the mainland, and the drone will be remotely piloted back to the district hospital. Technically, for the distance between Nkhata Bay and Likoma, the maximum cargo load is $4 \mathrm{~kg}$. But for a shorter distance between Likoma and Chizumulu, it can go up to $6 \mathrm{~kg}$.

Despite the thorough safety controls from the operation team, several interview participants of this field study reported witnessing adverse flight events, such as the so-called "hard landing" due to technical or human errors, as well as crashes of drones. In one of these incidents, it was reported that the drone lost control mid-air and crashed. As described by some of the technical staff as well as villagers living on the island, who were at or nearby the operation site when it occurred, the villagers saw a drone falling from the sky with fire and had a team run towards it with fire extinguishers. Although the villagers were informed that the drone might fall on trees or in the lake, and that it would unlikely injure them if they kept distance from the operation areas, they expressed fear of seeing a flying object with fire for the first time in their lives. Some associated the fire with witches or God, others worried about the fire spreading around and damage the island. During an interview with the local community members involved in this project, participants expressed concerns about drone safety, insisting that the operators and the DCA must take responsibility to ensure safety before allowing the drone to take off; and that if accidents happen frequently, then the drone is just "too dangerous". Participants raised concerns also regarding cargo safety, referring to situations in which a drone is crashed and the samples it carries are not packaged or sealed properly; and that since some of the samples carried by drones are infectious, such as TB sputum, then the leakage or spillage of these samples may fall over during drone accidents, potentially spreading infections around the island.

\section{ETHICAL ANALYSIS}

The aid sector represents considerable potentials for economic development of the tech industry, which is eager to search varied partners to project a green image of "tech for good" to the public [16][17]. Humanitarian and development work offer a golden opportunity of "killing two birds with one stone" - as one interview participant vividly put - they enable the tech industry to associate their image with humanitarian causes, while allowing for tests of their products on a large scale in countries where need is profound and regulation is relaxed. From the ethical perspective, this dual-purpose approach is precarious for two reasons: 1) introduction of new technology to humanitarian assistance and development programs can have unexpected consequences to the vulnerable populations involved, which calls for critical considerations when initiating innovation in the aid sector [12][13]; and 2) although the culture of taking risks and accepting failure is mainstream in innovation, such attitude may not suit the humanitarian and development contexts, where fundamental principles are derived from moral imperatives of humanity, impartiality, neutrality and independence [17][18]. In the context of this case study, this dual-purpose approach can be examined through the lens of what brought drones to Malawi and what drones brought to Malawi, to help unpack the importance of responsible riskbenefit assessments when introducing novel technology and developing innovation policies in the aid sector.

\section{A. What Brought Drones to Malawi?}

First of all, Malawi is faced with pressing public health challenges. In general, there are HIV/AIDS, TB and malaria, among other less prevalent diseases including measles, polio and hepatitis. For disease control and patient management purposes, biological samples including, e.g., sputum for TB, blood samples for HIV and malaria, and histology samples for cancer, need to be routinely collected and tested. The most common samples that need to be analyzed and tested in the lab are early infant diagnosis samples and viral load samples, both related to HIV/AIDS and must be monitored on a regular basis. Take viral load samples for example: if those who are already on antiretroviral therapies still have high blood viral load, then a process called enhanced adherence counselling must be provided during a three-month period. Therefore, the turnaround time of lab samples is critical in providing timely treatment plans and patient care. It might look as though the challenges are on an individual level, but it is in fact on a population level, as in the absence of accurate lab results, health workers in remote health facilities can only treat patients based on their experience. This affects not only the local Malawians, but also those from neighboring countries, such as Mozambique, who cross borders to seek medical treatment in Malawi.

Secondly, in terms of infrastructure barriers, there are areas in Malawi that are impassable during rainy seasons due to flooding. For these hard-to-reach areas, since 2011, there is an international organization providing a reliable and efficient transport system to the $\mathrm{MoH}$, which helps collect and deliver lab samples using motorbikes. This solution, however, does not apply to the islands on the other side of Lake Malawi. Due to a lack of resources on the islands, where paved roads do not exist and electricity is a persistent problem, the health centers and clinics do not have sufficient lab facilities to test samples locally and, thus, depend heavily on the mainland. However, in the lakeshore areas, the only existing mode of transportation is the native boat called "ilala" coming from Mozambique, which runs twice a week and takes about 6-8 hours each way. In the case of lab samples, the small islands need to send them first to the larger island, and then the samples from both islands are sent to the mainland, which takes about 5-8 weeks on average. When the health workers cannot bring the samples in person to the mainland, it would take additional weeks for them to find a passenger who travels on the boat to help bring the samples. The samples need to be stored in a cooler, and if anything goes wrong, e.g., if the ice packs burst, then the samples have to be discarded as they are no longer viable. In this process, samples are at a high risk of getting lost due to the lack of trained skills of the involved passengers. 
Thirdly, Malawi has an exceptionally "drone friendly" regulatory environment - from the various Ministries involved at the central level of the government down to the districts and local authorities. Additionally, Malawi has a largely free airspace where only a few flights per day are present, allowing relatively easy air traffic management. When drones came to Malawi, the GoM was interested in the opportunity and eager to know how Malawi could benefit from drones. Although at the onset of the project, there was no pre-existing regulation in Malawi particularly applicable to drones, the GoM was willing to facilitate drone activities in Malawi by providing operators with administrative supports. As long as the drone operators are technically capable, are compliant with the regulatory requirements, can fulfil safety obligations, and have robust operational practices, the DCA would grant them flight permissions. In 2017, with the support of its partners, the DCA started exploring how they could adopt the drone technology and establish regulations that would suit the specific context of Malawi. It took the DCA nearly two years to finally complete the draft regulation governing unmanned aircrafts, which was then submitted to the Ministry of Justice for approval [19]. However, as the top priority of the DCA is manned aircrafts, as is the case with most aviation authorities in the world, it is still a long way to go with regards to integrating drones into the existing airspace of Malawi.

Last but not least, and most important of all, with the assistance of its long-term in-country partner E, the GoM established the Malawi Drone Corridor in mid-2017, which is the first of its kind [3][20]. The Drone Corridor offers an integrated airspace for any organization wishing to test drone flights in Malawi, making drone operations significantly easier and smoother than any other county in the world. Since its inauguration, it has received over 15 drone operators from all over the world, including public and private organizations as well as drone manufacturers and service providers. These operators have done tests in both aerial mapping and medical deliveries, some also tested digital tracking systems, and unmanned traffic management systems. Although $\mathrm{E}$ is not involved in funding, planning or managing individual drone projects, they are helpful in sharing knowledge, fostering collaboration, and maintaining general management of the Corridor, which has become a gateway to Malawi for the global drone community. As the first international organization interested in using drones in Malawi to assist humanitarian and development work, $\mathrm{E}$ is also involved in strengthening local capacity by providing technical support to the DCA to establish its own unmanned aircraft regulations. In addition to the Drone Corridor, E recently launched another initiative - the Malawi Drone and Data Academy, to further strengthen local technical capacity building [21]. The hope is to, in the long run, roll out drone projects in Malawi or in Africa at large, using local personnel to improve the sustainability and scalability of local drone operations.

Overall, Malawi's pressing public health needs, significant logistical challenges for LMD, favorable regulatory environment, and prior experience with drones through the Drone Corridor, as well as the local population's high level of comfort with drones, all made Malawi a unique testing ground for drones [20]. In the context of this case study, the donor's funding preference and Malawi's health and logistical needs are well aligned, making Malawi a perfect operational environment to carry out drone testing activities. This match between drones and Malawi is seen by many stakeholders participated in the interviews during this filed study, especially those from the health sector, as "killing two birds with one stone" - carrying out drone testing activities in an ideal environment Malawi offers, on the one hand, while helping address some of the health challenges Malawi faces, on the other hand.

\section{B. What Drones Brough to Malawi?}

According to the interviewed stakeholders, the long-term vision between the GoM and the project lead was to run the drone operations long enough in Malawi with regular deliveries to demonstrate the utility and suitability of the approach, and to learn about how to integrate drones into the health supply chain system of Malawi, utilizing existing health personnel and facilities without building new structure. There seems to be a general expectation from the GoM that, after the initial phase of operation, not only would routine drone deliveries be provided in the identified area of operations, but they would also be scaled up in other remote areas of Malawi. The GoM also seems to be expectant of committed local capacity building activities on the technical front, such that eventually they would be able to run the drone operations by "having trained locals press a few buttons", with less dependence on the donors and the drone operators - who all "come from outside" and "do what they want, and not what we want".

From the technical perspective, the project was seen as successful. It was beyond showcasing the state-of-the-art of the drone technology, where the drone can vertically take-off and land and can fly BVLOS. Additionally, it was also demonstrated that, at its current level of development, the drone can fly multiple times a day between different locations, and can carry cargos bi-directionally. Furthermore, it was proven that the integration of drones in the local airspace of Malawi was feasible, and that it was possible to coordinate with the DCA using practical and simple solutions such as a WhatsApp group. The biggest technical challenge seems to be the highly unreliable mobile network across Malawi, where only intermittent service is available - sometimes even when operating near a cell tower of a known network provider. In particular, according to the drone pilots and ground staff, over Lake Malawi, there is no network coverage. As a result, when the drone flies over the lake, there is always about ten minutes where communication gets lost. This has caused drone accidents both previously with other operators and in this project. Poor communication infrastructure poses serious safety risks to humans and the environment, challenging the scalability of drone operations in Malawi.

From the health impact perspective, the project achieved its intended outcome with respect to lab sample transportation, and consequently, there has been a noticeable improvement in patient management. During the pilot operation, the samples were collected and delivered in time, and the test results were returned to the local health facilities in time, ensuring timely diagnosis and treatment which was previously unattainable with the boat. For the local health personnel on the islands, despite concerns regarding safety, resource and capacity, the drones helped resolve some of their immediate problems and gave them a certain sense of relief. However, for the District Health Officers at the district level and the $\mathrm{MoH}$ at the central level, since to date, every drone project carried out has "ended in a crash", there seems to be a general skepticism regarding how realistic the drone operation can truly be. Based on the knowledge and experience gained thus far, they seem to have 
readjusted their expectations towards using drones in Malawi, while continuing to look for effective solutions to address the health system challenges they are faced with.

From the benefit sharing perspective, the GoM demands all the drone manufacturers and service providers who come to Malawi to help facilitate local capacity, in addition to testing their own drones, in order to enable the GoM to consistently serve the underserved in the long run. As local capacity building and knowledge transfer has always been a prerequisite of the GoM, it was expected that the projects should train the local health workers to, e.g., receive and reload cargos, or even charge the drone batteries, such that they could learn and assume more technical responsibility in drone operations in the future. Following this guidance, some of the drone operators organized workshops through E, others offered hands-on trainings to young Malawians. The GoM also envisions to eventually train local Malawians to become licensed drone pilots. Reality, however, suggests that few locals have been trained so far, and that those who have been trained do not yet have a job related to drones. This is because all the drone projects coming to Malawi to date are in the trial phase - they do not stay and cannot create jobs in a real sense. There seems to be a recurring hope, each time a new operator comes in, that if the testing activities can succeed and be scaled up in Malawi, then the trainings to locals may be expanded and jobs for the trained locals may be created.

The medical cargo drone case is multifaceted. There is the issue of cost, of maturity of the technology, of certainty of a business case in the sense that there will be a return on what has been invested - all is largely unknown or unproven to date. Presently, drone operations are primarily funded by drone manufacturers who are interested in testing their technology or in advancing their R \& D, and typically these activities end up being a trail or a test. In the context of this project, the drone manufacturer and the donor agency partnered with each other as a result of mutual interest, jointly sharing investment as well as risks. To systematically assess the value creation of drones and measure the actual health impacts, the $\mathrm{MoH}$ would need to have at least one project that is fully functional and that is funded for a long-enough time. The DCA would then be able to issue the drone operators a registration certificate that they have envisioned of implementing. However, if the return is unknown or business case is unproven, then it is hard to justify spending and consequently difficult to secure funds - as donors hesitate to invest large amount of funds on something that is essentially uncertain. As a result, this becomes a dead-end, and a general attitude of "having someone else test it first and figure things out" exists within the global humanitarian drone community.

\section{CONCLUSION}

As this case study illustrates, drones offer an alternative option of transporting smaller payloads across shorter distances at higher frequency, potentially complementing traditional health logistic solutions to address the LMD challenge in resources-limited contexts. Notably, although drones are not the ultimate silver bullet, there is a compelling story to tell when it is about delivering medicines, saving lives, and helping improve health outcomes [20]. This, to a large extent, makes development one of the largest growth sectors for cargo drones [2]. While the public may be encouraged by the potentials of "drones for good", and implementers may be excited to use innovative technologies in their operation, governments need to ensure that drone- supported health systems can indeed make a difference in a sustainable manner, and that populations in need can actually gain improved access to the health services and products as so promised [20]. In this sense, what is urgently needed for the aid sector is finding a balance between allowing access to new technology for the most vulnerable on the one hand, and maintaining neutrality and independence in assessing the necessity, scope and degree of innovation on the other hand.

\section{NoTE}

* Due to space constraints, the methodology used for data collection in the field study and its subsequent analysis is not set out in this paper. The author welcomes any queries in this regard through direct correspondence.

\section{ACKNOWLEDGMENT}

In developing the ideas presented in this paper, the author received insightful feedback from Dr. Markus Christen (University of Zurich, Switzerland), and Dr. Matthew Hunt (McGill University, Canada), to whom she is immensely indebted. She is also grateful to Dr. Heather Collister for assistance with the interview data, and local stakeholders for fact validation, during the data analysis process. Her deepest gratitude goes to the project partners who supported her in the fieldwork, without whom this case study would not have been conceived in the first place.

\section{REFERENCES}

[1] K. B. Sandvik, K. Lohne, "The Rise of the Humanitarian Drone: Giving Content to an Emerging Concept," Millennium: Journal of International Studies, Vol. 43, no. 1, pp.145-164, DOI: https://doi.org/10.1177/0305829814529470, 2014.

[2] FSD, "Drones in Humanitarian Action: A guide to the use of airborne systems in humanitarian crises," FSD, 2016, available at: http://drones.fsd.ch/wp-content/uploads/2016/11/Drones-inHumanitarian-Action.pdf, accessed on: 02 September 2020.

[3] M. Eichleay, E. Evens, K Stankevitz, C Parker, "Using the Unmanned Aerial Vehicle Delivery Decision Tool to Consider Transporting Medical Supplies via Drone," Global Health: Science and Practice Journal, vol. 7, no. 4, pp. 500-506, DOI: https://doi.org/10.9745/GHSP-D-19-00119, 2019.

[4] Zipline, "Global Public Health," Zipline, available at: https://flyzipline.com/solutions/global-public-health/, accessed on: 02 September 2020.

[5] C. Mauluka, When the Drone Flies! Rethinking Communication Models in the Context of Innovations, Journal of Development Communication, vol. 30, no. 2, pp. 82-91, 2019.

[6] M. R. Dickey, "UPS Partners with Drone Startup Matternet for Medical Sample Delieveries," Tech Crunch, 2019, available at: https://techcrunch.com/2019/03/26/ups-partners-with-dronestartup-matternet-for-medical-sample-deliveries/, accessed on: 02 September 2020.

[7] J. Porter, "Google's Wing Drones Approved to Make Public Deliveries in Australia," The Verge, 2019, available at: https://www.theverge.com/2019/4/9/18301782/wing-dronedelivery-google-alphabet-canberra-australia-public-launch, accessed on: 02 September 2020.

[8] USAID Global Health Supply Chain Program-Procurement and Supply Management, "Unmanned Aerial Vehicles Landscape Analysis: Applications in the Development Context," Chemonics International Inc., 2017, available at: https://www.ghsupplychain.org/sites/default/files/201706/GHSC PSM UAV\%20Analysis final.pdf, accessed on: 02 September 2020. 
[9] A. Choi-Fitzpatrick, "Drones for Good: Technological Innovations, Social Movements, and the State," Journal of International Affairs, vol. 68, no. 1, pp. 19-36, 2014.

[10] C. Chen, 'Humanitarian Technology: Taking the 'Human' out of Humanitarianism?" RSIS Commentary, no. 154, 2019, available at: https://www.rsis.edu.sg/rsis-publication/nts/humanitariantechnology-taking-the-human-out-of-humanitarianism/\#.X2X2i2w01g, accessed on: 02 September 2020.

[11] J. C. Chow, "The Case for Humanitarian Drones," OpenCanada, 2012, available at: https://www.opencanada.org/features/the-casefor-humanitarian-drones/, accessed on: 02 September 2020.

[12] N. Wang, "A Successful Story that Can Be Sold"? A Case Study of Humanitarian Use of Drones, Proceedings of 2019 IEEE International Symposium on Technology and Society (ISTAS), IEEE, DOI: https://doi.org/10.1109/ISTAS48451.2019.8938015, 2019.

[13] N. Wang, "We Live on Hope...": Ethical Considerations of Humanitarian Use of Drones in Post-Disaster Nepal", IEEE Technology and Society Magazine, vol. 39, no. 3, pp. 76-85, IEEE, DOI: https://doi.org/10.1109/MTS.2020.3012332, 2020.

[14] S. Truog, L Maxim, C Matemba, C Blauvelt, H Ngwira, A Makaya, S Moreira, E Lawrence, G Ailstock, A Weitz, M West, O Defawe, "Insights Before Flights: How Community Perceptions, Can Make or Break Medical Drone Deliveries," Drones, vol. 4, no. 51, pp. 1-13, DOI: http://doi.org/10.3390/drones4030051, 2020.

[15] UNICEF, "Malawi Tests First Unmanned Aerial Vehicle Flights for HIV Early Infant Diagnosis", UNICEF, available at: https://www.unicef.org/media/media_90462.html, accessed on: 02 September 2020.

[16] B. Meiches, "Non-human humanitarians," Review of International Studies, vol. 45, no. 1, pp. 1-19, DOI: https://www.doi:.prg/10.1017/S0260210518000281, 2019.

[17] K. B. Sandvik, "African Drone Stories," BEHEMOTH: A Journal on Civilisation, vol. 8 , no. 2, pp. 73-96, DOI: https://doi.org/10.6094/behemoth.2015.8.2.870, 2015.

[18] H. Slim, Humanitarian Ethics: A Guide to the Morality of Aid in War and Disaster, Oxford University Press, 2015.

[19] Global Drone Regualtions Database: http://www.droneregulations.info, accessed on 02 September, 2020.

[20] A. M. Knoblauch, S. de la Rosa, J. Sherman, C. Blauvelt, C. Matemba, L. Maxim, O. D. Defawe, "Bi-directional Drones to Strengthen Healthcare Provision: Experiences and Lessons from Madagascar, Malawi and Senegal," BMJ Global Health, vol. 4, no. 4, pp. 1-10, DOI: https://www.doi.org/10.1136/bmjgh-2019001541, 2019.

[21] UNICEF, "The African Drone and Data Academy in Malawi", $U N I C E F$, available at: https://www.unicef.org/malawi/africandrone-and-data-academy-malawi, accessed on: 02 September 2020 . 